\title{
Utilizing Contextually Relevant Terms in Bilingual Lexicon Extraction
}

\author{
Azniah Ismail \\ Department of Computer Science \\ University of York \\ York YO10 5DD UK \\ azniah@cs.york.ac.uk
}

\author{
Suresh Manandhar \\ Department of Computer Science \\ University of York \\ York YO10 5DD UK \\ sureshecs.york.ac.uk
}

\begin{abstract}
This paper demonstrates one efficient technique in extracting bilingual word pairs from non-parallel but comparable corpora. Instead of using the common approach of taking high frequency words to build up the initial bilingual lexicon, we show contextually relevant terms that co-occur with cognate pairs can be efficiently utilized to build a bilingual dictionary. The result shows that our models using this technique have significant improvement over baseline models especially when highestranked translation candidate per word is considered.
\end{abstract}

\section{Introduction}

Bilingual lexicons or dictionaries are invaluable knowledge resources for language processing tasks. The compilation of such bilingual lexicons remains as a substantial issue to linguistic fields. In general practice, many linguists and translators spend huge amounts of money and effort to compile this type of knowledge resources either manually, semiautomatically or automatically. Thus, obtaining the data is expensive.

In this paper, we demonstrate a technique that utilizes contextually relevant terms that co-occur with cognate pairs to expand an initial bilingual lexicon. We use unannotated resources that are freely available such as English-Spanish Europarl corpus (Koehn, 2005) and another different set of cognate pairs as seed words.

We show that this technique is able to achieve high precision score for bilingual lexicon extracted from non-parallel but comparable corpora. Our model using this technique with spelling similarity approach obtains 85.4 percent precision at 50.0 percent recall. Precision of 79.0 percent at 50.0 percent recall is recorded when using this technique with context similarity approach. Furthermore, by using a string edit-distance vs. precision curve, we also reveal that the latter model is able to capture words efficiently compared to a baseline model.

Section 2 is dedicated to mention some of the related works. In Section 3, the technique that we used is explained. Section 4 describes our experimental setup followed by the evaluation results in Section 5. Discussion and conclusion are in Section 6 and 7 respectively.

\section{Related Work}

Koehn and Knight (2002) describe few potential clues that may help in extracting bilingual lexicon from two monolingual corpora such as identical words, similar spelling, and similar context features. In reporting our work, we treat both identical word pairs and similar spelling word pairs as cognate pairs.

Koehn and Knight (2002) map 976 identical word pairs that are found in their two monolingual German-English corpora and report that 88.0 percent of them are correct. They propose to restrict the word length, at least of length 6 , to increase the accuracy of the collected word pairs. Koehn and Knight (2002) mention few related works that use different measurement to compute the similarity, such as longest common subsequence ratio (Melamed, 1995) and string edit distance (Mann 
and Yarowski, 2001). However, Koehn and Knight (2002) point out that majority of their word pairs do not show much resemblance at all since they use German-English language pair. Haghighi et al. (2008) mention one disadvantage of using edit distance, that is, precision quickly degrades with higher recall. Instead, they propose assigning a feature to each substring of length of three or less for each word.

For approaches based on contextual features or context similarity, we assume that for a word that occurs in a certain context, its translation equivalent also occurs in equivalent contexts. Contextual features are the frequency counts of context words occurring in the surrounding of target word $W$. A context vector for each $W$ is then constructed, with only context words found in the seed lexicon. The context vectors are then translated into the target language before their similarity is measured.

Fung and Yee (1998) point out that not only the number of common words in context gives some similarity clue to a word and its translation, but the actual ranking of the context word frequencies also provides important clue to the similarity between a bilingual word pair. This fact has motivated Fung and Yee (1998) to use tfidf weighting to compute the vectors. This idea is similar to Rapp (1999) who proposed to transform all co-occurrence vectors using log likelihood ratio instead of just using the frequency counts of the co-occurrences. These values are used to define whether the context words are highly associated with the $W$ or not.

Earlier work relies on a large bilingual dictionary as their seed lexicon (Rapp, 1999; Fung and Yee, 1998; among others). Koehn and Knight (2002) present one interesting idea of using extracted cognate pairs from corpus as the seed words in order to alleviate the need of huge, initial bilingual lexicon. Haghighi et al. (2008), amongst a few others, propose using canonical correlation analysis to reduce the dimension. Haghighi et al (2008) only use a small-sized bilingual lexicon containing 100 word pairs as seed lexicon. They obtain 89.0 percent precision at 33.0 percent recall for their EnglishSpanish induction with best feature set, using topically similar but non-parallel corpora.

\section{The Utilizing Technique}

Most works in bilingual lexicon extraction use lists of high frequency words that are obtained from source and target language corpus to be their source and target word lists respectively. In our work, we aim to extract a high precision bilingual lexicon using different approach. Instead, we use list of contextually relevant terms that co-occur with cognate pairs.

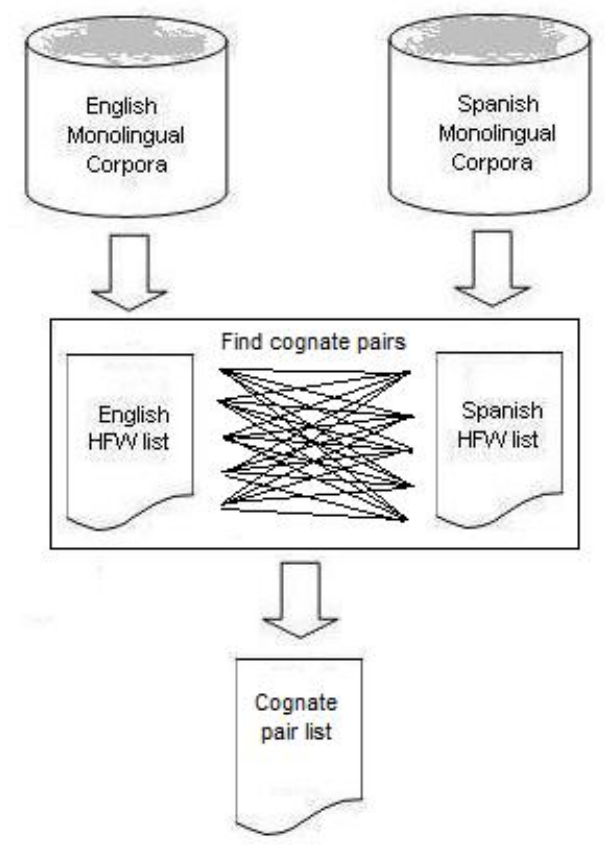

Figure 1: Cognate pair extraction

These cognate pairs can be derived automatically by mapping or finding identical words occur in two high frequency list of two monolingual corpora (see Figure 1). They are used to acquire list of source word $W_{s}$ and target word $W_{t}$. $W_{s}$ and $W_{t}$ are contextually relevant terms that highly co-occur with the cognate pairs in the same context. Thus, log likelihood measure can be used to identify them.

Next, bilingual word pairs are extracted among words in these $W_{s}$ and $W_{t}$ list using either context similarity or spelling similarity. Figure 2 shows some examples of potential bilingual word pairs, of $W_{s}$ and $W_{t}$, co-occurring with identical cognate pairs of word 'civil'.

As we are working on English-Spanish language pair, we extract bilingual lexicon using string edit distance to identify spelling similarity between $W_{s}$ 
and $W_{t}$. Figure 3 outlines the algorithm using spelling similarity in more detail.

Using the same $W_{s}$ and $W_{t}$ lists, we extract bilingual lexicon by computing the context similarity between each $\left\{W_{s}, W_{t}\right\}$ pair. To identify the context similarity, the relation between each $\left\{W_{s}, W_{t}\right\}$ pair can be detected automatically using a vector similarity measure such as cosine measure as in (1). The $A$ and $B$ are the elements in the context vectors, containing either zero or non-zero seed word values for $W_{s}$ and $W_{t}$, respectively.

$$
\text { Cosine similarity }=\cos (\theta)=\frac{A \times B}{\|A\| \times\|B\|}
$$

The cosine measure favors $\left\{W_{s}, W_{t}\right\}$ pairs that share the most number of non-zero seed word values. However, one disadvantage of this measure is that the cosine value directly proportional to the actual $W_{s}$ and $W_{t}$ values. Even though $W_{s}$ and $W_{t}$ might not closely correlated with the same set of seed words, the matching score could be high if $W_{s}$ or $W_{t}$ has high seed word values everywhere. Thus, we transform the context vectors from real value into binary vectors before the similarity is computed. Figure 4 outlines the algorithm using context similarity in more detail.

In the algorithm, after the $W_{s}$ and $W_{t}$ lists are obtained, each $W_{s}$ and $W_{t}$ units is represented by their context vector containing log likelihood (LL) values of contextually relevant words, occurring in the seed lexicon, that highly co-occur with the $W_{s}$ and $W_{t}$ respectively. To get this context vector, for each $W_{s}$ and $W_{t}$, all sentences in the English or Spanish corpora containing the respective word are extracted to form a particular sub corpus, e.g. sub corpus society is a collection of sentences containing the source word society.

Using window size of a sentence, the LL value of term occurring with the word $W_{s}$ or $W_{t}$ in their respective sub corpora is computed. Term that is highly associated with the $W_{s}$ or $W_{t}$ is called contextually relevant term. However, we consider each term with LL value higher than certain threshold (e.g. threshold $\geq 15.0$ ) to be contextually relevant. Contextually relevant terms occurring in the seed lexicon are used to build the context vector for the

\begin{tabular}{|rl|}
\hline \multicolumn{2}{|c|}{ CIVIL } \\
$\begin{array}{r}\text { society } \\
\text { rights }\end{array}$ & $\begin{array}{l}\text { sociedad } \\
\text { derechos }\end{array}$ \\
development & desarrollo \\
cooperation & cooperación \\
military & militar \\
dialogue & diálogo \\
representatives & representantes \\
democracy & democracia \\
international & internacional \\
forces & fuerzas \\
government & gobierno \\
security & seguridad \\
participation & participación \\
conflict & conflicto \\
freedoms & libertades \\
aviation & aviación \\
protection & protección \\
organisations & organizaciónes \\
organisation & organización \\
administration & administración \\
& \\
\hline
\end{tabular}

Figure 2: Bilingual word pairs are found within context of cognate word civil
1. Automatic cognate pairs derivation Obtain high frequency lists from both monolingual corpora $\Rightarrow H F W_{S}$ and $H F W_{T}$ lists.

For all pairs taken from the $H F W_{S}$ and $H F W_{T}$ lists, find identical cognate pairs, $C$.

\section{Source word and target word list} For every $C$ :

Extract all sentences containing $C \Rightarrow>S u b$ corpora $C$ Using window size of a sentence for Sub corpora $C$, compute the log likelihood of all terms occurring with word $C=>L L_{C}$

From $L L_{C}$, obtain 100 highly-ranked contextually relevant terms in respective language $\Rightarrow W_{s}$ and $W_{t}$

\section{Spelling similarity measure}

For every pair of $W_{s}$ and $W_{t}$ :

Compute similarity using edit distance

$\Rightarrow$ SpellingSim $\left(W_{s}, W_{t}\right)$

Obtain all matched bilingual word pairs above threshold or highest-ranked word pairs.

Figure 3: Utilizing technique with spelling similarity 


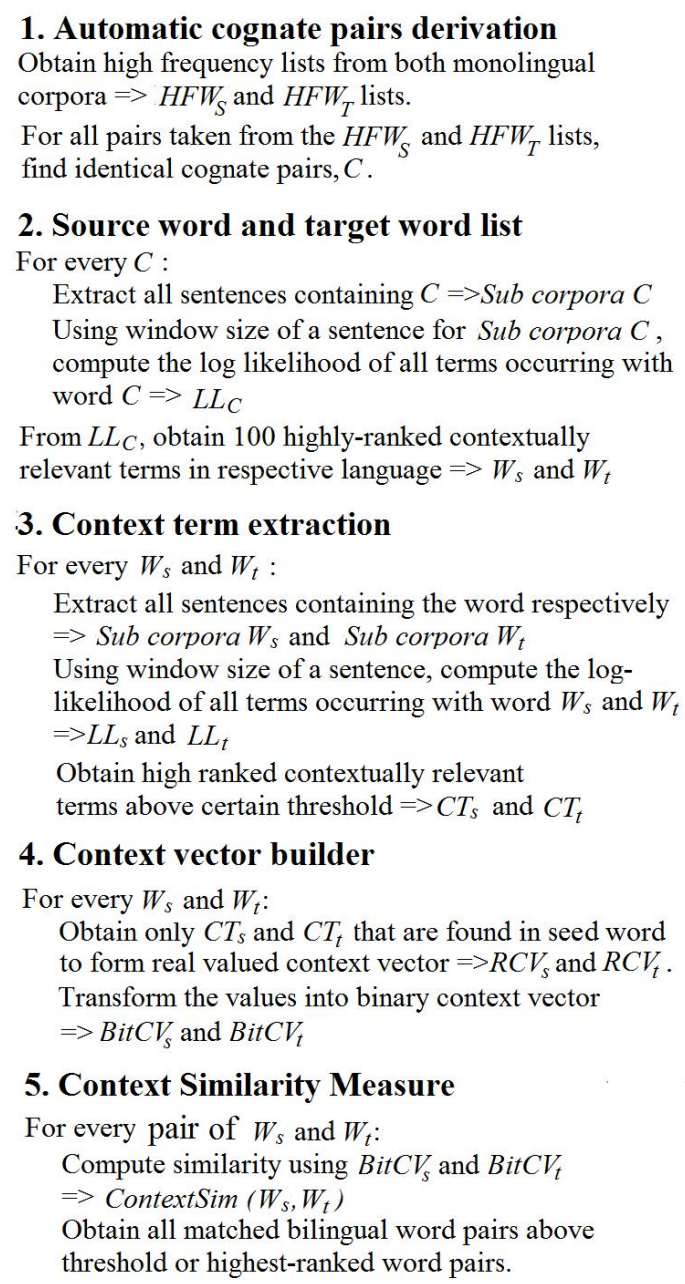

\section{Context term extraction}

For every $W_{s}$ and $W_{t}$ :

Extract all sentences containing the word respectively

$\Rightarrow$ Sub corpora $W_{s}$ and Sub corpora $W_{t}$

Using window size of a sentence, compute the log-

likelihood of all terms occurring with word $W_{s}$ and $W_{t}$

$\Rightarrow L L_{s}$ and $L L_{t}$

Obtain high ranked contextually relevant

terms above certain threshold $\Rightarrow C T_{s}$ and $C T_{t}$

\section{Context vector builder}

For every $W_{s}$ and $W_{t}$ :

Obtain only $C T_{s}$ and $C T_{t}$ that are found in seed word to form real valued context vector $\Rightarrow R C V_{s}$ and $R C V_{t}$.

Transform the values into binary context vector

$\Rightarrow$ BitCV $_{s}$ and BitCV

5. Context Similarity Measure

For every pair of $W_{S}$ and $W_{t}$ :

Compute similarity using $\mathrm{BitCV}_{s}$ and $\mathrm{BitCV}_{t}$

$\Rightarrow$ ContextSim $\left(W_{s}, W_{t}\right)$

Obtain all matched bilingual word pairs above

threshold or highest-ranked word pairs.

Figure 4: Utilizing technique with context similarity

$W_{s}$ or $W_{t}$ respectively. For example, word participation and education occurring in the seed lexicon are contextually relevant terms for source word society. Thus, they become elements of the context vector. Then, we transform the context vectors, from real value into binary, before we compute the similarity with cosine measure.

\section{Experimental Setup}

\subsection{Data}

For source and target monolingual corpus, we derive English and Spanish sentences from parallel Europarl corpora (Koehn, 2005).

- We split each of them into three parts; year
1996 - 1999, year 2000 - 2003 and year 2004 - 2006.

- We only take the first part, about 400k sentences of Europarl Spanish (year 1996 - 1999) and 2nd part, also about 400k from Europarl English (year 2000 - 2003). We refer the particular part taken from the source language corpus as $S$ and the other part of the target language corpus as $T$.

This approach is quite common in order to obtain non-parallel but comparable (or same domain) corpus. Examples can be found in Fung and Cheung (2004), followed by Haghighi et al. (2008). For corpus pre-processing, we only use sentence boundary detection and tokenization on raw text. We decided that large quantities of raw text requiring minimum processing could also be considered as minimal since they are inexpensive and not limited. These should contribute to low or medium density languages for which annotated resources are limited. We also clean all tags and filter out stop words from the corpus.

\subsection{Evaluation}

We extracted our evaluation lexicon from Word Reference $^{*}$ free online dictionary . For this work, the word types are not restricted but mostly are content words. We have two sets of evaluation. In one, we take high ranked candidate pairs where $W_{s}$ could have multiple translations. In the other, we only consider highest-ranked $W_{t}$ for each $W_{s}$. For evaluation purposes, we take only the top 2000 candidate ranked-pairs from the output. From that list, only candidate pairs with words found in the evaluation lexicon are proposed. We use F1-measure to evaluate proposed lexicon against the evaluation lexicon. The recall is defined as the proportion of the high ranked candidate pairs. The precision is given as the number of correct candidate pairs divided by the total number of proposed candidate pairs.

\subsection{Other Setups}

The following were also setup and used:

- List of cognate pairs We obtained 79 identical cognate pairs from the

*from website http: / / www. wordreference.com 
top 2000 high frequency lists of our $S$ and $T$ but we chose 55 of these that have at least 100 contextually relevant terms that are highly associated with each of them.

\section{- Seed lexicon}

We also take a set of cognate pairs to be our seed lexicon. We defined the size of a small seed lexicon ranges between 100 to $1 \mathrm{k}$ word pairs. Hence, our seed lexicon containing 700 cognate pairs are still considered as a smallsized seed lexicon. However, instead of acquiring this set of cognate pairs automatically, we compiled the cognate pairs from a few Learning Spanish Cognates websites ${ }^{\dagger}$. This approach is a simple alternative to replace the 10-20k general dictionaries (Rapp, 1999; Fung and McKeown, 2004) or automatic seed words (Koehn and Knight, 2002; Haghighi et al., 2008). However, this approach can only be used if the source and target language are fairly related and both share lexically similar words that most likely have same meaning. Otherwise, we have to rely on general bilingual dictionaries.

\section{- Stop list}

Previously (Rapp, 1999; Koehn and Knight, 2002; among others) suggested filtering out commonly occurring words that do not help in processing natural language data. This idea sometimes seem as a negative approach to the natural articles of language, however various studies have proven that it is sensible to do so.

\section{- Baseline system}

We build baseline systems using basic context similarity and spelling similarity features.

\section{Evaluation Results}

For the first evaluation, candidate pairs are ranked after being measured either with cosine for context similarity or edit distance for spelling similarity. In this evaluation, we take the first 2000 of $\left\{W_{s}, W_{t}\right\}$ candidate pairs from the proposed lexicon where $W_{s}$ may have multiple translations or multiple $W_{t}$. See Table 1.

\footnotetext{
${ }^{\dagger}$ such as http://www.colorincolorado.org and http: //www. language-learning-advisor.com
}

\begin{tabular}{|l|r|r|r|r|r|}
\hline Setting & $\mathbf{P}_{0} .1$ & $\mathbf{P}_{0} .25$ & $\mathbf{P}_{0} .33$ & $\mathbf{P}_{0} .5$ & Best-F1 \\
\hline ContextSim $(\boldsymbol{C S})$ & 42.9 & 69.6 & 60.7 & 58.7 & 49.6 \\
SpellingSim $(\boldsymbol{S S})$ & 90.5 & 74.2 & 69.9 & 64.6 & 50.9 \\
\hline
\end{tabular}

(a) from baseline models

\begin{tabular}{|l|r|r|r|r|r|}
\hline Setting & $\mathbf{P}_{0} .1$ & $\mathbf{P}_{0} .25$ & $\mathbf{P}_{0} .33$ & $\mathbf{P}_{0} .5$ & Best-F1 \\
\hline E-ContextSim $(\boldsymbol{E C S})$ & 78.3 & 73.5 & 71.8 & 64.0 & 51.2 \\
E-SpellingSim $(\boldsymbol{E S S})$ & 95.8 & 75.6 & 71.8 & 63.4 & 51.5 \\
\hline
\end{tabular}

(b) from our proposed models

Table 1: Performance of baseline and our model for top 2000 candidates below certain threshold and ranked

\begin{tabular}{|l|r|r|r|r|r|}
\hline Setting & $\mathbf{P}_{0} .1$ & $\mathbf{P}_{0} .25$ & $\mathbf{P}_{0} .33$ & $\mathbf{P}_{0} .5$ & Best-F1 \\
\hline ContextSim-Top1 $(\boldsymbol{C S T})$ & 58.3 & 61.2 & 64.8 & 55.2 & 52.6 \\
SpellingSim-Top1 $(\boldsymbol{S S T})$ & 84.9 & 66.4 & 52.7 & 34.5 & 37.0 \\
\hline
\end{tabular}

(a) from baseline models

\begin{tabular}{|l|r|r|r|r|r|}
\hline Setting & $\mathbf{P}_{0} .1$ & $\mathbf{P}_{0} .25$ & $\mathbf{P}_{0} .33$ & $\mathbf{P}_{0} .5$ & Best-F1 \\
\hline E-ContextSim-Top1 $(\boldsymbol{E C S T})$ & 85.0 & 81.1 & 79.7 & 79.0 & 57.1 \\
E-SpellingSim-Top1 $(\boldsymbol{E S S T})$ & 100.0 & 93.6 & 91.6 & 85.4 & 59.0 \\
\hline
\end{tabular}

(b) from our proposed models

Table 2: Performance of baseline and our model for top 2000 candidates of top 1

Using either context or spelling similarity approach on $S$ and $T$ (labeled ECS and ESS respectively), our models achieved about 51.2 percent of best F1 measure. Those are not a significant improvement with only 1.0 to 2.0 percent error reduction over the baseline models (labeled $C S$ and $S S$ ).

For the second evaluation, we take the first 2000 of $\left\{W_{s}, W_{t}\right\}$ pairs where $W_{s}$ may only have the highest ranked $W_{t}$ as translation candidates (See Table 2 ). This time, both of our models (with context similarity and spelling similarity, labeled ECST and ESST respectively) yielded almost 60.0 percent of best $\mathrm{F} 1$ measure. It is noted that using ESST alone recorded a significant improvement of 20.0 percent in the F1 score compared to SST baseline model. ESST obtained 85.4 percent precision at 50.0 percent recall. Precision of 79.0 percent at 50.0 percent recall is recorded when using ECST. However, the ECST has not recorded a significant difference over CST baseline model (57.1 and 52.6 percent respectively) in the second evaluation. The overall performances, represented by precision scores for different 


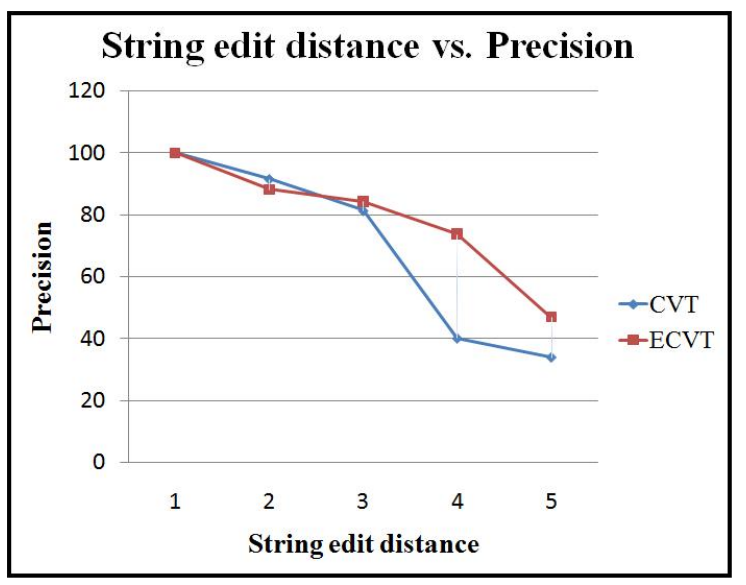

Figure 5: String Edit Distance vs. Precision curve

range of recalls, for these four models are illustrated in Appendix A.

It is important to see the inner performance of the ECST model with further analysis. We present a string edit distance value $(E D v)$ vs. precision curve for ECST and CST in Figure 5 to measure the performance of the ECST model in capturing bilingual pairs with less similar orthographic features, those that may not be captured using spelling similarity.

The graph in Figure 5 shows that even though $C S T$ has higher precision score than ECST at EDv of 2, it is not significant (the difference is less than 5.0 percent) and the spelling is still similar. On the other hand, precision for proposed lexicon with $E D v$ above 3 (where the $W_{s}$ and the proposed translation equivalent $W_{t}$ spelling becoming more dissimilar) using ECST is higher than CST. The most significant difference of the precision is almost 35.0 percent, where ECST achieved almost 75.0 percent precision compared to CST with 40.0 percent precision at $E D v$ of 4 . It is followed by ECST with almost 50.0 percent precision compared to CST with precision less than 35.0 percent, offering about 15.0 percent precision improvement at $E D v$ of 5.

\section{Discussion}

As we are working on English-Spanish language pair, we could have focused on spelling similarity feature only. Performance of the model using this feature usually record higher accuracy otherwise they may not be commonly occurring in a corpus. Our models with this particular feature have recorded higher F1 scores especially when considering only the highest-ranked candidates.

We also experiment with context similarity approach. We would like to see how far this approach helps to add to the candidate scores from our corpus $S$ and $T$. The other reason is sometimes a correct target is not always a cognate even though a cognate for it is available. Our ECST model has not recorded significant improvement over CST baseline model in the F1-measure. However, we were able to show that by utilizing contextually relevant terms, ECST gathers more correct candidate pairs especially when it comes to words with dissimilar spelling. This means that ECST is able to add more to the candidate scores compared to CST. Thus, more correct translation pairs can be expected with a good combination of ECST and ESST.

The following are the advantages of our utilizing technique:

- Reduced errors, hence able to improve precision scores.

- Extraction is more efficient in the contextual boundaries (see Appendix B for examples).

- Context similarity approach within our technique has a potential to add more to the candidate scores.

Yet, our attempt using cognate pairs as seed words is more appropriate for language pairs that share large number of cognates or similar spelling words with same meaning. Otherwise, one may have to rely on bilingual dictionaries.

There may be some possible supporting strategies, which we could use to help improve further the precision score within the utilizing technique. For example, dimension reduction using canonical correlation analysis (CCA), resemblance detection, measure of dispersion, reference corpus and further noise reduction. However, we do not include a reranking method, as we are using collection of cognate pairs instead of a general bilingual dictionary. Since our corpus $S$ and $T$ is in similar domain, we might still not have seen the potential of this technique in its entirety. One may want to test the technique with different type of corpora for future works. 
Nevertheless, we are still concerned that many spurious translation equivalents were proposed because the words actually have higher correlation with the input source word compared to the real target word. Otherwise, the translation equivalents may not be in the boundaries or in the corpus from which translation equivalents are to be extracted. Haghighi et al (2008) have reported that the most common errors detected in their analysis on top 100 errors were from semantically related words, which had strong context feature correlations. Thus, the issue remains. We leave all these for further discussion in future works.

\section{Conclusion}

We present a bilingual lexicon extraction technique that utilizes contextually relevant terms that cooccur with cognate pairs to expand an initial bilingual lexicon. We show that this utilizing technique is able to achieve high precision score for bilingual lexicon extracted from non-parallel but comparable corpora. We demonstrate this technique using unannotated resources that are freely available.

Our model using this technique with spelling similarity obtains 85.4 percent precision at 50.0 percent recall. Precision of 79.0 percent at 50.0 percent recall is recorded when using this technique with context similarity approach. We also reveal that the latter model with context similarity is able to capture words efficiently compared to a baseline model. Thus, we show contextually relevant terms that cooccur with cognate pairs can be efficiently utilized to build a bilingual dictionary.

\section{References}

Cranias, L., Papageorgiou, H, and Piperidis, S. 1994. A matching technique in Example-Based Machine Translation. In International Conference On Computational Linguistics Proceedings, 15th conference on Computational linguistics, Kyoto, Japan.

Diab, M., and Finch, S. 2000. A statistical word-level translation model for comparable corpora. In Proceedings of the Conference on Content-based multimedia information access (RIAO).

Fung, P., and Cheung, P. 2004. Mining very non-parallel corpora: Parallel sentence and lexicon extraction via bootstrapping and EM. In Proceedings of the 2004
Conference on Empirical Method in Natural Language Processing (EMNLP), Barcelona, Spain.

Fung, P., and Yee, L.Y. 1998. An IR Approach for Translating New Words from Nonparallel, Comparable Texts. In Proceedings of COLING-ACL98, Montreal, Canada, 1998.

Fung, P., and McKeown, K. 1997. Finding Terminology Translations from Non-parallel Corpora. In The 5th Annual Workshop on Very Large Corpora, Hong Kong, Aug 1997.

Haghighi, A., Liang, P., Berg-Krikpatrick, T., and Klein, D. 2008. Learning bilingual lexicons from monolingual corpora. In Proceedings of The ACL 2008, June 15 -20 2008, Columbus, Ohio

Koehn, P. 2005. Europarl: a parallel corpus for statistical machine translation. In MT Summit

Koehn, P., and Knight , K. 2001. Knowledge sources for word-level translation models. In Proceedings of the Conference on Empirical Methods in Natural Language Processing (EMNLP).

Koehn, P., and Knight , K. 2002. Learning a translation lexicon from monolingual corpora. In Proceedings of ACL 2002, July 2002, Philadelphia, USA, pp. 9-16.

Rapp, R. 1995. Identifying word translations in nonparallel texts. In Proceedings of ACL 33, pages 320322.

Rapp, R. 1999. Automatic identification of word translations from unrelated English and German corpora. In Proceedings of ACL 37, pages 519-526. 
Appendix A. Precision scores with different recalls

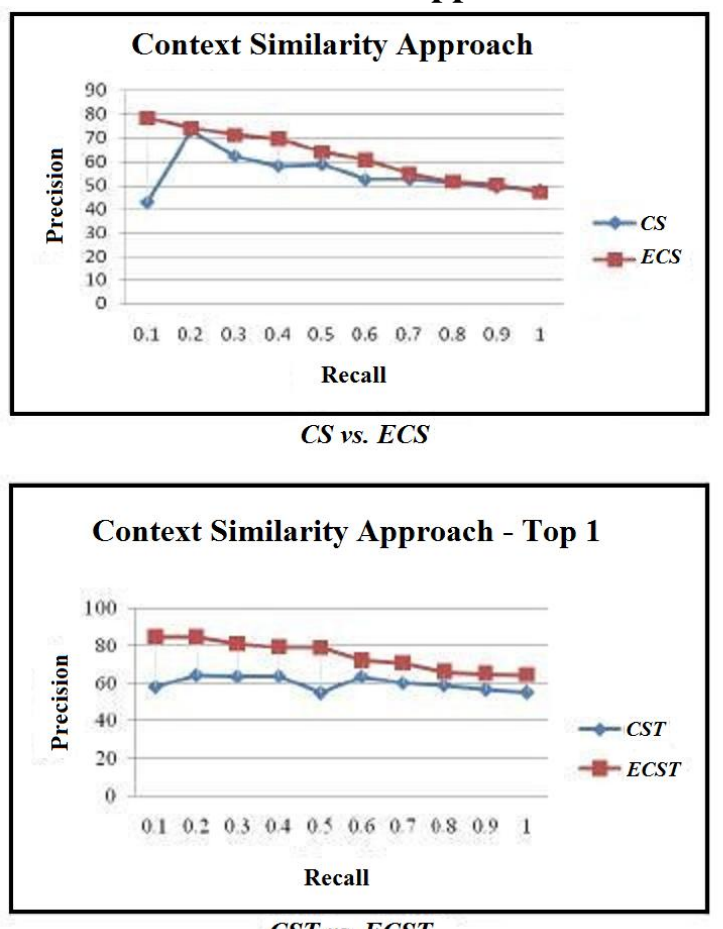

CST vs. ECST

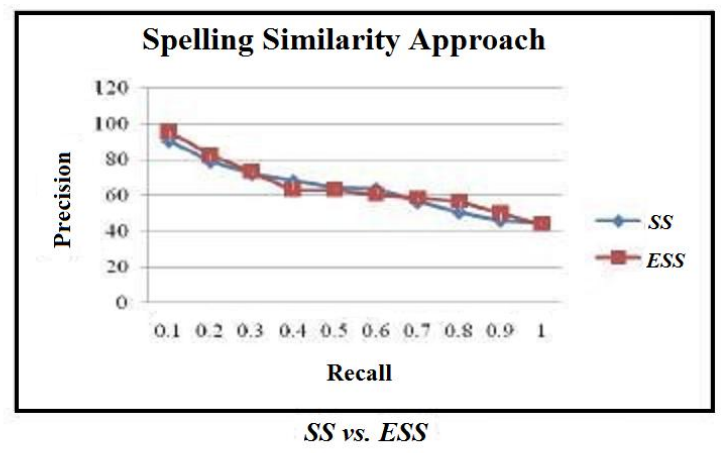

Spelling Similarity Approach -Top 1

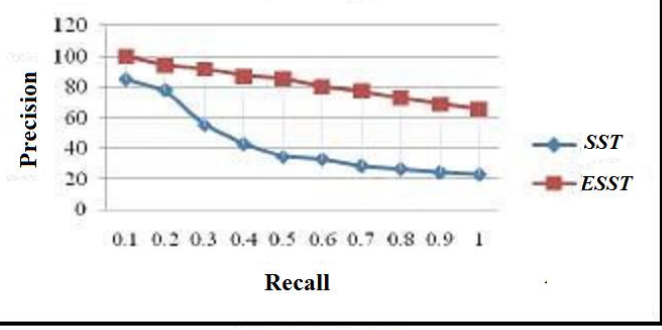

SST vs. ESST

Appendix B. Some examples of effective extraction via utilizing technique

\begin{tabular}{|c|c|c|c|c|c|c|}
\hline \multirow[b]{2}{*}{ Source } & \multirow[b]{2}{*}{ Target } & \multicolumn{2}{|c|}{ ECVT } & \multicolumn{2}{|c|}{$\mathrm{CV}$} & \multirow[b]{2}{*}{ Rank } \\
\hline & & Candidate found & Sim. value & Candidate found & Sim. value & \\
\hline clause & clausula & clausula & 0.402015126 & $\begin{array}{l}\text { autentica } \\
\text { fortalecimiento } \\
\text { economico } \\
\text { respeto } \\
\text { vigor } \\
\text { clausula }\end{array}$ & \begin{tabular}{|l|}
0.447213595 \\
0.430331483 \\
0.412478956 \\
0.40824829 \\
0.402015126 \\
0.402015126 \\
\end{tabular} & $\begin{array}{l}1 \\
2 \\
<> \\
<> \\
<> \\
<>\end{array}$ \\
\hline pillar & pilar & pilar & 0.547722558 & $\begin{array}{l}\text { daramente } \\
\text { pilar } \\
\text { basada } \\
\text { comercial } \\
\text { iniciado } \\
\text { exterior } \\
\text { agricola }\end{array}$ & $\begin{array}{c}0.632455532 \\
\mathbf{0 . 5 4 7 7 2 2 5 5 8} \\
0.53935989 \\
0.516397779 \\
0.516397779 \\
0.478091444 \\
0.447213595\end{array}$ & $\begin{array}{l}1 \\
2 \\
3 \\
4 \\
4 \\
5 \\
6\end{array}$ \\
\hline state & estado & estado & 0.433012702 & $\begin{array}{l}\text { derecho } \\
\text { estado } \\
\text { respeto }\end{array}$ & $\mid$\begin{tabular}{l|}
0.43519414 \\
0.433012702 \\
0.412478956
\end{tabular} & $\begin{array}{c}1 \\
2 \\
<>\end{array}$ \\
\hline confidence & confianza & confianza & 0.424264069 & $\begin{array}{c}\text { errores } \\
\text { desarrollo } \\
\text { haberse } \\
\text { demuestran } \\
\text { deficiencias } \\
\text { confianza }\end{array}$ & $\begin{array}{l}0.447213595 \\
0.447213595 \\
0.447213595 \\
0.447213595 \\
0.447213595 \\
0.424264069\end{array}$ & $\begin{array}{l}1 \\
1 \\
1 \\
1 \\
1 \\
1\end{array}$ \\
\hline welfare & bienestar & bienestar & 0.40824829 & $\begin{array}{c}\text { hubiera } \\
\text { bienestar }\end{array}$ & $\begin{array}{r}0.500000000 \\
0.40824829\end{array}$ & $\begin{array}{c}1 \\
<>\end{array}$ \\
\hline
\end{tabular}

